\title{
SOUND TRANSMISSION THROUGH CYLINDRICAL SHELL STRUCTURES EXCITED BY BOUNDARY LAYER PRESSURE FLUCTUATIONS
}

\author{
Yvette Y. Tang ${ }^{1}$ \\ National Research Council \\ MS 463, NASA Langley Research Center, Hampton, VA 23681 \\ Richard J. Silcox ${ }^{2}$ and Jay H. Robinson ${ }^{3}$ \\ Structural Acoustical Branch \\ MS 463, NASA Langley Research Center, Hampton, VA 23681
}

\begin{abstract}
This paper examines sound transmission into two concentric cylindrical sandwich shells subject to turbulent flow on the exterior surface of the outer shell. The interior of the shells is filled with fluid medium and there is an airgap between the shells in the annular space. The description of the pressure field is based on the cross-spectral density formulation of Corcos, Maestrello, and Efimtsov models of the turbulent boundary layer. The classical thin shell theory and the first-order shear deformation theory are applied for the inner and outer shells, respectively. Modal expansion and the Galerkin approach are used to obtain closed-form solutions for the shell displacements and the radiation and transmission pressures in the cavities including both the annular space and the interior. The average spectral density of the structural responses and the transmitted interior pressures are expressed explicitly in terms of the summation of the cross-spectral density of generalized force induced by the boundary layer turbulence. The effects of acoustic and hydrodynamic coincidences on the spectral density are observed. Numerical examples are presented to illustrate the method for both subsonic and supersonic flows.
\end{abstract}

\section{Introduction}

Sound transmission into the interior of aerospace vehicles such as subsonic and supersonic aircraft and launch vehicles has received significant attention recently since the noise levels in these vehicles exceed acceptable criteria. Past work has well defined the dominant noise transmission mechanisms for harmonic sources exciting this type of structure. However, much work remains to understand the dominant mechanism under boundary layer excitation. Therefore, there is a need for a better understanding of the interaction between temporally \& spatially random fluctuating pressure fields and elastic structures. A number of

1. Research Associate, Member AIAA.

2. Assistant Branch Head, Member AIAA.

3. Research Engineer, Member AIAA. authors have published analyses of both the vibration response of elastic plate structures and the subsequent radiated sound pressure level induced by the turbulent boundary layer (TBL) pressure fluctuations $[3,5,6,8$, $9,16]$. Only a few papers have aimed at predicting the response and consequent noise radiation of isotropic shell structures subject to the turbulent flow due to the boundary layer $[1,7,10,12]$. The authors have studied sound transmission into cylindrical shells from an oblique incident plane sound wave for both single and two concentric cylindrical sandwich shells [13, 14]. Results have revealed that sandwich structures with honeycomb cores can offer an advantage for noise reduction over isotropic structures in the high frequency range and the double sandwich structures can produce an appreciable increase of transmission loss over single sandwich structures.

The objective of this paper is to develop an analytical model for sound transmission into two concentric cylindrical sandwich shells due to the TBL pressure fluctuations. The dynamic model for the problem under consideration is as follows: (1) the fluctuating pressures due to the TBL in the external flow adjacent to the surface of the outer shell cause the shell to vibrate; (2) these vibrations induce pressure fluctuations including radiation and transmission pressures in the annular space; (3) the transmitted pressures then excite the inner shell to vibrate; and (4) these vibrations generate noise in the interior cavity inside the shells. Based on this analysis, we require descriptions of the five fields: (1) the external pressure field acting on the shell wall induced by the TBL; (2) the vibration of the outer shell; (3) the annual cavity (airgap) pressure field; (4) the vibration of the inner shell; and (5) the interior cavity pressure field. The outer shell vibrations are assumed not to affect the TBL pressure field in the external flow and the radiation field from the outer shell is neglected.

\section{Pressure Fluctuation from the TBL}

A schematic of the system configuration is shown in Fig. 1, i.e., two concentric finite cylindrical shells with length $L$ and radii $R_{1}$ and $R_{2}$. The outer shell is 
excited by the pressure fluctuations of TBL on its exterior surface. Assume that the boundary layer is fully turbulent and the pressure field over the outer surface may be characterized as temporally stationary with spatially homogeneous statistics. Therefore, the pressure field may be expressed by a cross-correlation function that is decaying with spatial and time separation and is convected with the flow. The empirical models [2, 4, 9] for the TBL (Eq. (1)) show that the correlation function is dependent on the streamwise separation, $\xi=x_{1} \cong x_{2}$ and the crossflow separation, $\eta=R_{1}\left(\theta_{1} \cong \theta_{2}\right)$, where $x$ and $\theta$ denote the cylindrical coordinates along the longitudinal and circumferential directions, respectively. The Fourier transformation in time gives the spatial-frequency crossspectral density (CSD) as $S_{p}$

$S_{p}(\xi, \eta, \omega)=\Phi(\omega) A(\xi, \omega) B(\eta, \omega) e^{\mathrm{i} \omega \xi / U_{c}}$

where $\mathrm{i}=\rceil \cong 1, \omega$ is the frequency, $U_{c}$ is the convective velocity of the flow, and $\Phi$ is the power spectrum. Quantities $A$ and $B$ are the spatial correlation functions corresponding to $x$ and $\theta$ and are suggested to take the exponential decaying forms,

$$
A=\exp [-\alpha|\xi|], \quad B=\exp [-\beta|\eta|]
$$

A complete description of the $S_{p}$ requires the four quantities, $\Phi, U_{c}, \alpha$, and $\beta$, to be specified. In the following, we will define these quantities based on the Corcos, Maestrello, and Efimtsov models .

\subsection{Corcos model}

Corcos [2] suggested that in a convenient manner, the functions $A$ and $B$ as well as the dependence of $U_{c}$ on $\omega$ were inferred from the experiments of Willmarth $\&$ Wooldridge [15] as exponential forms. Therefore, the exponential coefficients $\alpha$ and $\beta$ take the following forms

$\alpha=\alpha^{\mathrm{C}} \omega / U_{c}, \quad \beta=\beta^{\mathrm{C}} \omega / U_{c}$

where $\alpha^{\mathrm{C}}$ and $\beta^{\mathrm{C}}$ are constant parameters determined by the experimental results. The typical range of the values is $\alpha^{\mathrm{C}}=0.11$ to 0.12 and $\beta^{\mathrm{C}}=0.7$ to 1.2 for a smooth rigid wall. Here, we assume $\alpha^{\mathrm{C}}=0.1$ and $\beta^{\mathrm{C}}=$ 0.77 .

\subsection{Maestrello model}

Only one of Maestrello's models is considered here in which the flow is assumed semi-frozen and it decays in space and time at a constant velocity $U_{c}$ [9]. After performing a comprehensive set of experimental results of wind tunnel testing, he defined an ensemble average of the cross correlation for the pressure fluctuation due to the TBL in which the effect of the Reynolds number and the boundary layer thickness was included. The Fourier transformation in time of the cross correlation of the pressure evaluates,

$$
\begin{gathered}
S_{p}(\xi, \eta, \omega)=\left\langle\overline{p^{2}}>\left(\delta / U_{e}\right) e^{\cong \mid \xi /\left(\alpha^{\mathrm{M}} \delta\right)} e^{\cong \mid \xi /\left(\beta^{\mathrm{M}} \delta\right)} \times\right. \\
\mathrm{e}^{\mathrm{i} \omega \xi / U_{c}} \lambda_{j=1}^{4} A_{j}^{\mathrm{M}} \exp \left[\cong K_{j}^{\mathrm{M}} \omega \delta / U_{c}\right]
\end{gathered}
$$

where $\delta$ is the boundary layer thickness and $U_{e}$ is the free stream velocity of the flow; $\alpha^{\mathrm{M}}=50 / \overline{C_{f} R_{\theta}}$ and $\beta^{\mathrm{M}}$ $=0.26$, and $\overline{C_{f} R_{\theta}}$ is the equivalent incompressible Reynolds number; parameters $A_{j}^{\mathrm{M}}$ and $K_{j}^{\mathrm{M}}$ are determined by the experiments and the values for $A_{1}^{\mathrm{M}}$ to $A_{4}^{\mathrm{M}}$ are $4.4 * 10^{\cong 2}, 7.5 * 10^{\cong 2}, \cong 9.3 * 10^{\cong 2}, \cong 2.5 * 10 \cong 2$ and for $K_{1}^{\mathrm{M}}$ to $K_{4}^{\mathrm{M}}$ are $5.78 * 10^{\cong 2}, 2.43 * 10^{\cong 1}, 1.12$, 11.57; the mean square value of the pressure fluctuation $\left\langle\overline{p^{2}}\right\rangle$ is related to the square of the wall shear stress $\tau_{w}^{2}$ by a proportional value, i.e., $\left\langle\overline{p^{2}}\right\rangle=12 \tau_{w}^{2}$ given by Maestrello and $\left\langle\overline{p^{2}}\right\rangle=4.8 \tau_{w}^{2}$ given by Willmarth and Wooldridge [15]. Equation (4) implies that the power spectrum $\Phi$ is

$$
\Phi(\omega)=\left\langle\overline{p^{2}}\right\rangle\left(\delta / U_{e}\right) \lambda_{j=1}^{4} A_{j}^{\mathrm{M}} \exp \left[\cong K_{j}^{\mathrm{M}} \omega \delta / U_{c}\right]
$$

and the exponential coefficients $\alpha$ and $\beta$ are

$\alpha=1 /\left(\alpha^{\mathrm{M}} \delta\right), \quad \beta=1 /\left(\beta^{\mathrm{M}} \delta\right)$

\subsection{Efimtsov model}

The reliability of the probability characteristics of wall pressure fluctuations of the TBL is largely determined by the quantity of reliable statistical data in the require ranges of Mach numbers, Reynolds numbers, and Strouhal numbers. However, under laboratory condition, it is impossible to cover the all these factors. Therefore, Efimtsov has performed a series of flight tests in the range of Mach numbers $0.41 \cong 2.1$ and measured the power spectra, spatial correlation, and convective velocity of the pressure fluctuations on the surface of an aircraft. He proposed the following formula for $\alpha$ and $\beta$ in Eq. (2) as following [4]

$\alpha=1 / \alpha^{\mathrm{E}}, \quad \beta=1 / \beta^{\mathrm{E}}$

where the expressions for $\alpha^{\mathrm{E}}$ and $\beta^{\mathrm{E}}$ are

$\alpha^{\mathrm{E}}=\delta\left[\left(\frac{\left(a_{1} S h\right)}{U_{c} / u_{\tau}}\right)^{2}+\frac{a_{2}^{2}}{S h^{2}+\left(a_{2} / a_{3}\right)^{2}}\right]^{\cong 1 / 2}$

$\beta^{\mathrm{E}}= \begin{cases}\delta\left[\frac{a_{4}^{2} S h^{2}}{U_{c}^{2} / u_{\tau}^{2}}+\frac{a_{5}^{2}}{S h^{2}+\left(a_{5} / a_{6}\right)^{2}}\right]^{\cong 1 / 2}, M<0.75 \\ \delta\left[\frac{a_{4}^{2} S h^{2}}{U_{c}^{2} / u_{\tau}^{2}}+a_{7}^{2}\right]^{\cong 1 / 2}, & M>0.95\end{cases}$

where $M$ is the free-stream Mach number. The average values of the empirical constants $a_{1}$ to $a_{7}$ are $0.1,72.8$, $1.54,0.77,548,13.5,5.66$. It can be shown that at high frequencies, these expressions correspond to a Corcos model with $\alpha^{\mathrm{C}}=0.1$ and $\beta^{\mathrm{C}}=0.7$. 
The convected velocity $U_{c}$, generally speaking, depends on frequency and spatial separations of the observation points, but the effect of the separation is neglected in this study. Two cases for $U_{c}$ are considered. The first one is that $U_{c}$ is related to the free stream velocity $U_{e}$ by a factor of proportionality and the factor decreases slightly with frequency varying from 0.6 to 0.8 [12]. It is approximated here by a value 0.65. The second one is using an empirical function for the $U_{e}$ suggested by Efimtsov [4]

$$
U_{c}=u_{\tau} a_{8} S h^{1 / 5}\left\{\left[1+\left(a_{9} S h\right)^{2}\right] /\left[1+\left(a_{10} S h\right)^{4}\right]\right\}^{1 / 10}
$$

where parameters $a_{8}$ to $a_{10}$ are $9.55,6.38 * 10^{\cong 4}$, $3.98 * 10^{\cong 3}$ and $S h=\omega \delta / u_{\tau}$. The friction velocity $u_{\tau}$ is related to the $U_{e}$ and skin-friction coefficient $c_{f}, u_{\tau}=U_{e}$ \rceil $c_{f} / 2$.

\section{Governing equations}

As shown in Fig. 1, the two concentric shells are surrounded by fluid media and there is an airgap between them. The pressure fluctuations from the TBL excite the exterior surface of the outer shell. The mass density and sound speed for the external, annular, and internal fluid media are denoted by $\left\{\rho_{0}, c_{0}\right\},\left\{\rho_{1}, c_{1}\right\}$, and $\left\{\rho_{2}\right.$, $\left.c_{2}\right\}$, respectively. Each of the sandwich shells may be specified as a honeycomb core with face sheets that can be isotropic, orthotropic, or laminated fiber-reinforced composite materials. The inner shell is a thin shell so that the classical thin shell theory is used. However, the outer shell is a thick shell, therefore the effect of the shear deformation and rotation can not be neglected especially at high frequencies [13]. The first-order shear deformation shell theory is applied for vibration of the outer shell. The shell motion due to the turbulence is assumed sufficiently small so that linear theory may be employed and the pressure fields are also assumed to be linear. To develop solutions, we assume that the radiation pressure does not affect the TBL pressure loading of the external flow. Based on the above descriptions, we can write the governing equations as follows including the vibration equations for the outer and inner shells and the acoustic wave equations for the annular and internal fluids.

The governing equations for an infinite cylindrical shell in terms of displacement components in the middle surface along the radial, axial and circumferential directions have been shown in the paper [13]. These equations can still be used here since no boundary conditions were introduced. The displacement components along the axial and circumferential directions are solved for in terms of the displacement components in the radial direction. Taking temporal Fourier transformation, the governing equations become

$L_{1}(\omega)\left[w_{1}\right]=p_{1} \cong p_{0}, L_{2}(\omega)\left[w_{2}\right]=p_{2} \cong p_{1}$ where $w_{1}$ and $w_{2}$ are the radial displacements and $L_{1}$ and $\mathrm{L}_{2}$ are frequency dependent differential operators corresponding to the outer and inner shells, respectively and $p_{0}, p_{1}$, and $p_{2}$ are the acoustic pressures corresponding to the external, annular, and internal fluid fields, respectively.

The total fluctuating pressure in the external fluid $p_{0}$ is composed of the random fluctuating pressure $p$ induced by the TBL and the radiation pressure $p^{\prime}$, i.e., $p$ $p_{0}=p+p^{\prime}$. The radiation pressure term $p^{\prime}$ is neglected in this analysis. Therefore, $p_{0} p$. With this assumption, only the linear acoustic wave equations for the annular and internal fluid need to be considered. The Fourier transformations of the wave equations give Helmholz equations

$\nabla^{2} p_{1}+k_{1}^{2} p_{1}=0, \quad \nabla^{2} p_{2}+k_{2}^{2} p_{2}=0$

where $k_{1}^{2}=\omega^{2} / c_{1}^{2}, \quad k_{2}^{2}=\omega^{2} / c_{2}^{2}, \quad \nabla^{2}=\diamond(r \diamond / \diamond r)+$ $\left(\diamond^{2} / \vec{\theta}\right) / r^{2}+\diamond^{2} / \bullet^{2}$, and $\{r, \theta, x\}$ represent cylindrical coordinates along the radial, circumferential, and longitudinal directions, respectively.

The flexible boundary conditions applied at the inner surface of the outer shell and the outer surface of the inner shell relate the pressure $p_{1}$ to the shell displacements $w_{1}$ and the inner wall displacement $w_{2}$

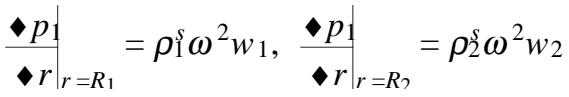

where $\rho_{1}^{S}$ and $\rho_{2}^{S}$ are the mass densities of the outer and inner shells, respectively. At the interior surface of the inner wall

$$
\frac{\bullet p_{2}}{\bullet r}=\rho_{2}^{S} \omega^{2} w_{2} \text {, at } r=R_{2}
$$

applies.

It is assumed that the shells are simply-supported at both ends and therefore the boundary conditions are

$w_{1}=w_{2}=\frac{\diamond^{2} w_{1}}{\iota^{2}}=\frac{\diamond^{2} w_{2}}{\iota^{2}}=0$, at $x=0, L$

The gradients of the pressures $p_{1}$ and $p_{2}$ are zero at both ends

$\frac{\bullet p_{1}}{\bullet x}=\frac{\diamond p_{2}}{\bullet x}=0$, at $x=0, L$

in which an assumption is made that the end plates are acoustically hard.

\section{Closed-form Solution}

In order to obtain the solutions, the technique used here is the modal expansion analysis and Galerkin approach. From boundary conditions, Eq. (14), we assume that the displacement components $w_{1}$ and $w_{2}$ 
can be expanded in terms of the orthogonal base functions $X_{m n}$

$$
\begin{aligned}
& w_{1}=\prod_{m=1, n=0}^{\infty} w_{1 m n} X_{m n}(x, \theta) \\
& w_{2}=\prod_{m=1, n=0}^{\infty} w_{2 m n} X_{m n}(x, \theta)
\end{aligned}
$$

where $w_{1 m n}$ and $w_{2 m n}$ are unknown modal coefficients and

$X_{m n}=\sin (m \pi x / L) \cos (n \theta)$

As will be shown later, the exclusion of the $\sin (n \theta)$ functions from the basis functions $X_{m n}$ does not limit the generality of the solution. Then we can expand the pressure fluctuations of the TBL $p$ as

$p=\prod_{m=1, n=0}^{\infty} p_{m n} X_{m n}(x, \theta)$

where the generalized forces $p_{m n}$ are defined by

$p_{m n}=\varepsilon_{n} /(\pi L) \times\left[\int_{0}^{L} \int_{0}^{2 \pi} p(x, \theta, \omega) X_{m n}(x, \theta) d x d \theta\right]$

in which $\varepsilon_{n}$ are the Neumann factors, $\varepsilon_{n}=1$ for $n=0$ and $\varepsilon_{n}=2$ otherwise.

Similarly, the annular acoustic pressure $p_{1}$ which satisfies Eq. (11) can be expanded as

$p_{1}=\lambda_{m=1, n=0}^{\infty}\left[p_{11 m n} J_{n}\left(\sigma_{1 m} r\right)+p_{12 m n} Y_{n}\left(\sigma_{1 m} r\right)\right] X_{m n}(20)$

where $J_{n}$ and $Y_{n}$ are the first and second kinds of Bessel functions of order $n, \sigma_{1 m}^{2}=k_{1}^{2} \cong(m \pi / L)^{2}$, and $p_{11 m n}$ and $p_{12 m n}$ are underdetermined modal coefficients. In order to obtain a convergent solution at $r=0$, the interior acoustic pressure $p_{2}$ which is the solution of Eq. (11) can be expanded as

$p_{2}=\lambda_{m=1, n=0}^{\infty} p_{2 m n} J_{n}\left(\sigma_{2 m} r\right) X_{m n}(x, \theta)$

Similarly, $\quad \sigma_{2 n}^{2}=k_{2}^{2} \cong(m \pi / L)^{2}$ and $p_{2 m n}$ are yetunderdetermined modal coefficients.

Inspection of Eqs. (20) and (21) reveals that the pressures $p_{1}$ and $p_{2}$ do not satisfy the boundary conditions, Eq. (15). To meet this need, we expand the orthogonal functions $X_{m n}$,

$X_{m n}=\prod_{j=0, j \neq m}^{\infty} \frac{\varepsilon_{j}}{\pi} \frac{m\left[1 \cong(\cong 1)^{m \cong j}\right]}{m^{2} \cong j^{2}} Y_{j n}(x, \theta)$

where $Y_{j n}$ are orthogonal base functions of $p_{1}$ and $p_{2}$ which satisfy the boundary condition Eqs. (15)

$Y_{j n}=\cos (j \pi x / L) \cos (n \theta)$
The unknown modal coefficients $w_{1 m n}, w_{1 m n}$, $p_{11 m n}, p_{12 m n}$, and $p_{2 m n}$ can be obtained by solving the coupled equations, Eqs. (10), (12), and (13), simultaneously. The closed form expressions for these quantities are given as follows,

$\left\{w_{1 m n}, w_{2 m n}, p_{11 m n}, p_{12 m n}, p_{2 m n}\right\}=$
$\left\{\widetilde{w}_{1 m n}, \widetilde{w}_{2 m n}, \widetilde{p}_{11 m n}, \widetilde{p}_{12 m n}, \widetilde{p}_{2 m n}\right\} p_{m n}$

where

$\widetilde{w}_{1 m n}=\cong \mathrm{i}\left[J_{12 d} Y_{11 d} Z_{22}^{S J J} \cong J_{11 d} Y_{12 d} Z_{22}^{S J Y}\right] /(\omega \Delta)$
$\widetilde{w}_{2 m n}=\cong \mathrm{i} J_{12 d} Y_{12 d} Z_{12}^{J Y} /(\omega \Delta)$
$\widetilde{p}_{11 m n}=\left(\mathrm{i} \rho_{1} \omega / \sigma_{1 m}\right) Y_{12 d} Z_{22}^{S J Y} / \Delta$
$\widetilde{p}_{12 m n}=\cong\left(\mathrm{i} \rho_{1} \omega / \sigma_{1 m}\right) J_{12 d} Z_{22}^{S J J} / \Delta$
$\widetilde{p}_{2 m n}=\cong \mathrm{i} J_{12 d} Y_{12 d} Z_{12}^{J Y} \rho_{2} \omega /\left(\sigma_{1 m} J_{22 d} \Delta\right)$

in which

$J_{11}=J_{n}\left(\sigma_{1 m} R_{1}\right), \quad J_{11 d}=J_{n}^{\prime}\left(\sigma_{1 m} R_{1}\right)$

$J_{12}=J_{n}\left(\sigma_{1 m} R_{2}\right), \quad J_{12 d}=J_{n}^{\prime}\left(\sigma_{1 m} R_{2}\right)$

$J_{22}=J_{n}\left(\sigma_{2 m} R_{2}\right), \quad J_{22 d}=J_{n}^{\prime}\left(\sigma_{2 m} R_{2}\right)$

$Y_{11}=Y_{n}\left(\sigma_{1 m} R_{1}\right), \quad Y_{11 d}=Y_{n}^{\prime}\left(\sigma_{1 m} R_{1}\right)$

$Y_{12}=Y_{n}\left(\sigma_{1 m} R_{2}\right), \quad Y_{12 d}=Y_{n}^{\prime}\left(\sigma_{1 m} R_{2}\right)$

and

$Z_{11}^{S J}=Z_{11}^{S} \cong\left(\mathrm{i} \omega \rho_{1} / \sigma_{1 m}\right)\left(J_{11} / J_{11 d}\right)$

$Z_{11}^{S Y}=Z_{11}^{S} \cong\left(\mathrm{i} \omega \rho_{1} / \sigma_{1 m}\right)\left(Y_{11} / Y_{11 d}\right)$

$Z_{12}^{J Y}=\left(\mathrm{i} \omega \rho_{1} / \sigma_{1 m}\right)\left(J_{11} / J_{11 d} \cong Y_{11} / Y_{11 d}\right)$

$Z_{22}^{S J J}=Z_{22}^{S} \cong \mathrm{i} \omega\left[\rho_{2} J_{22} /\left(\sigma_{2 m} J_{22 d}\right)+\rho_{1} J_{12} /\left(\sigma_{1 m} J_{12 d}\right)\right]$

$Z_{22}^{S J Y}=Z_{22}^{S} \cong \mathrm{i} \omega\left[\rho_{2} J_{22} /\left(\sigma_{2 m} J_{22 d}\right)+\rho_{1} Y_{12} /\left(\sigma_{1 m} Y_{12 d}\right)\right](27 \mathrm{e})$

$\Delta=J_{12 d} Y_{11 d} Z_{11}^{S Y} Z_{22}^{S J J} \cong J_{12 d} Y_{11 d} Z_{11}^{S J} Z_{22}^{S J Y}$

and $Z_{11}^{S}$ and $Z_{22}^{S}$ are the modal impedances of the outer and inner shells, respectively.

\section{Cross-spectral Density}

It is necessary to evaluate the modal-spectral density $S_{p_{m n}}$ of the generalized TBL forcing function before we discuss the cross-spectral density (CSD) of the structural response and transmission sound pressure. The $S_{p_{m n}}$ embodies the correlation function describing the boundary-layer pressures and is defined in terms of the normalized-pressure CSD,

$$
\begin{aligned}
S_{p_{m n}} & =\left[\varepsilon_{n} /(\pi L)\right]^{2} \int_{0}^{L} d x_{1} \int_{0}^{L} d x_{2} \int_{0}^{2 \pi} d \theta_{1} \int_{0}^{2 \pi} d \theta_{2} S_{p}[\xi, \eta, \omega] \\
& \sin \left[m \pi x_{1} / L\right] \sin \left[m \pi x_{2} / L\right] \cos \left[n \theta_{1}\right] \cos \left[n \theta_{2}\right]
\end{aligned}
$$

Substitute Eqs. (1) and (2) into above equation, then utilize the periodic properties of the integral along 
circumferential direction $\theta$ yielding a closed form for this integral,

$S_{p_{m n}}=\operatorname{Re}\left|4 \Phi(\omega) \varepsilon_{n} I_{\xi m} I_{\theta n}\left(\pi L R_{1}\right)^{\cong 1}\left(\alpha^{\prime} \beta\right)^{\cong 1}\right|$

where Re|.| is the real part of the argument,

$$
\begin{aligned}
I_{\xi m}= & \left\{1+\left[m \pi /\left(\alpha^{\prime} L\right)\right]^{2}+2\left[1 \cong(\cong 1)^{m} e^{\cong \alpha^{\prime} L}\right] \times\right. \\
& \left.(m \pi)^{2}\left(\alpha^{\prime} L\right)^{\cong 3}\right\} \times\left\{1+\left[m \pi /\left(\alpha^{\prime} L\right)\right]^{2}\right\}^{\cong 2}
\end{aligned}
$$

$I_{\theta n}=\left[1 \cong(\cong 1)^{n} e^{\cong \beta R_{1} \pi}\right] \times\left\{1+\left[n /\left(\beta R_{1}\right)\right]^{2}\right\}^{\cong 1}$

where $\alpha^{\prime}=\alpha+\mathrm{i} \omega / U_{c}$ and $\alpha$ and $\beta$ are determined by Eqs. (3), (6), and (7). The product of $I_{\xi m}$ and $I_{\eta_{n}}$ gives the joint acceptance of the pressure field and the mode concerned which was introduced in the paper [11]. It can be shown from Eq. (30a) that under the condition of the hydrodynamic coincidence, $\omega=U_{c} m \pi / L$, the denominator becomes very small which results in a large value for the modal-spectral density function, $S_{p_{m n}}$. When $\alpha=0.11 \omega / U_{c}$ and $\beta=0.6 \omega / U_{c}$, equation (29) is reduced to the results derived by Rattayya and Junger [12].

Structural vibratory response is of interest because of its direct application to the analysis of stress and fatigue life of structures. Also an increasing concern for interior noise generated by exterior flows in modern high-speed transport systems, both on the ground and in the air, requires a thorough understanding of the mechanics of this structural acoustics problem. In the following we will discuss the normalized average spectral density (NASD) of the structural response and the transmitted interior pressure. Based on the analysis [12], the modal spectral density of the shell displacements and the transmitted interior sound pressure are defined as follows

$$
\begin{aligned}
& S_{w_{1 m n}}=\Phi(\omega)\left|\widetilde{w}_{1 m n}\right|^{2} S_{p_{m n}} \\
& S_{w_{2 m n}}=\Phi(\omega)\left|\widetilde{w}_{2 m n}\right|^{2} S_{p_{m n}} \\
& S_{p_{2 m n}}=\Phi(\omega)\left|\tilde{p}_{2 m n}\right|^{2} S_{p_{m n}}
\end{aligned}
$$

This equation implies that the mean response is not a function of circumferential position, and therefore independent of the coordinate $\theta$ selected. This is consistent with the selection of only the $\cos (n \theta)$ basis functions in $X_{m n}$ in Eq. (17), where it is assumed the orthogonal set of $\sin (n \theta)$ functions would yield identical results.

The average spectral density of the shell displacements $w_{1}$ and $w_{2}$ and the sound pressure $p_{2}$ in the interior over the shell area is independent of the location observed and boundary conditions at the both ends. Therefore, the final forms for the average spectral density are given as following

$$
\left\{S_{w_{1}}, S_{w_{2}}, S_{p_{2}}\right\}=\lambda_{m=1, n=0}^{\infty}\left\{S_{w_{1 m n}}, S_{w_{2} m n}, S_{p_{2 m n}}\right\} / 2
$$

In order to predict the sound transmission characteristics of the system, we consider the NASD which is defined by

$$
\left\{\bar{S}_{w_{1}}, \bar{S}_{w_{2}}, \bar{S}_{p_{2}}\right\}=\left\{S_{w_{1}}, S_{w_{2}}, S_{p_{2}}\right\} / \Phi(\omega)
$$

\section{Results and Discussion}

In the numerical analysis, the material properties and geometry for the shells are the same as those in paper [14] but with a finite length $L=30 \mathrm{~m}$ : The outer shell consists of titanium face sheets and titanium honeycomb core and the inner shell consists of four layer laminated cross-ply graphite/epoxy face sheets and aluminum honeycomb core. The fiber orientation for the inner shell is $\left\{90^{\circ}, 0^{0}, 90^{\circ}, 0^{\circ}\right.$, honeycomb core, $\left.0^{0}, 90^{\circ}, 0^{0}, 90^{\circ}\right\}$ with axial direction measured from the exterior surface of the inner shell. The radius and wall thickness are $R_{1}=1.88 \mathrm{~m}$ and $h_{1}=5.079 \mathrm{~cm}$ for the outer shell and $R_{2}=1.84 \mathrm{~m}$ and $h_{2}=0.635 \mathrm{~cm}$ for the inner shell. The face sheets are made from the same material and with the same thickness for each shell. The thickness ratio of the core and the total for each shell is 0.84 . The material properties of the face sheet and the core are given in Table. 1 and 2. The structural loss factor is 0.01. Because there is no experimental data about TBL pressure for the considered problem, the data used here are taken from Maestrello's paper [9] for the free stream velocity $U_{e}$, the thickness and displacement of the boundary layer $\delta$ and $\delta^{*}$, the friction coefficient $c_{f}$, and the equivalent incompressible Reynolds number $\overline{C_{f} R_{\theta}}$. Two representative cases are considered: one is subsonic with Mach number $M=$ 0.67 at sea level $\left(\rho_{2}=1.2243 \mathrm{~kg} / \mathrm{m}^{3}, c_{2}=340.445 \mathrm{~m} / \mathrm{s}\right)$ and another is supersonic with Mach number $M=1.42$ at cruising flight altitude with the internal pressure set to that at $10,000 \mathrm{ft} \quad\left(\rho_{2}=0.9041 \mathrm{~kg} / \mathrm{m}^{3}, \quad c_{2}=\right.$ $328.558 \mathrm{~m} / \mathrm{s}$ ). The mass density and sound speed for the annular fluid medium are the same as those for the internal fluid medium. In the numerical analysis, the interior pressure considered $p_{2}$ is at $r=R_{2}$.

Figure 2 shows the coefficients $\alpha$ and $\beta$ defined in Eqs. (3), (6), and (7) versus frequency for $M=0.67$ and $M=1.42$ among Corcos, Maestrello, and Efimtsov models. Both $\alpha$ and $\beta$ increase linearly with frequency in the Corcos model while they are constants in Maestrello's model. It can be seen that as the frequency approaches infinity, $\alpha$ and $\beta$ in the Efimtsov model will tend towards those in Corcos model.

Figure 3 shows the NASD of the shell displacements $w_{1}$ and $w_{2}$ and the interior pressure $p_{2}$ defined in Eq. (33) for $M=0.67$. There are three phenomena which lead to the peak values in the NASD so that sound can be transmitted efficiently at these frequencies. The first one is the resonance frequencies of the coupled system at which the peaks in the NASD 
are present among all figures. The second one is the acoustic coincidence frequencies (near $4000 \mathrm{~Hz}$ and $8500 \mathrm{~Hz})$ at which the bending wave speed of the inner shell associated with an infinite plate with the same material and thickness equals the acoustic wave speed of the fluid. Sharp peaks in the NASD of $w_{2}$ and $p_{2}$ are seen at the acoustic coincidences, as shown in Fig. 3(b) and 3(c). The last one is the hydrodynamic coincidence frequencies at which the bending wave speed of the inner shell equals the convected velocity of the flow. These results demonstrate that the response of the outer shell is affected slightly by the resonance modes and hydrodynamic coincidences. However, beside the influenced of the resonance modes and hydrodynamic coincidences, the response of the inner shell is strongly affected by the acoustic coincidences. The influence of the resonance modes and the acoustic and hydrodynamic coincidences on the NASD of $p_{2}$ is significant. Noise can be transmitted very efficiently by the resonant modes above the hydrodynamic and acoustic coincidences, as shown in Fig. 3(c). Comparing three curves in each of the figures shows that at low frequency, Maestrello's and Efimtsov's models give close results. However, at frequencies greater than 500 $\mathrm{Hz}$, all the models tend to merge into one curve. The consistency between the Corcos model and Efimtsov model is not surprising due to the convergence of the exponential coefficients $\alpha$ and $\beta$ of the Efimtsov model to those of the Corcos model at higher frequencies. It is surprising that Maestrello's model tends toward the other models although the coefficients $\alpha$ and $\beta$ are defined differently, as shown in Fig. 2. This illustrates that the NASD is not sensitive to the selection of the exponential coefficients especially at higher frequencies in this particular study. Since there are no significant differences among the three models, we will present the results based on the Corcos model in the following examples.

Figure 4 shows the effect of the Mach number on the NASD of $w_{1}, w_{2}$, and $p_{2}$ for $M=0.67$ and 1.42 . The excitation and the acoustic properties of the fluids are not the same for the two cases, and therefore the characteristics of the coupled system are different. The different characteristics will result in differences in the resonances and the acoustic and hydrodynamic coincidences. From these figures, one can see that the hydrodynamic coincidences are not sensitive to this change and the NASD of $w_{1}$ increases with an increase of the Mach number slightly, as seen in Fig. 4a. However, the effect of the acoustic properties of the fluids on the acoustic coincidences is obvious and leads to the peaks being shifted. A significant effect of the Mach number on the resonances at which the peak values of the NASD of $p_{2}$ are shifted can be observed. The results illustrate that the effect of the Mach number on the NASD of the structural response is not obvious but is significant on the NASD of the interior pressure level.
Figure 5 shows the effect of $U_{c}$ on the NASD of $w_{1}, w_{2}$, and $p_{2}$ for $M=0.67$. The "formula" in the figures refers to the empirical function for $U_{c}$ defined in Eq. (9). The selection of $U_{c}$ only leads to the difference in the forcing field so that the hydrodynamic coincidences will be different for the two cases. These results illustrate that there is a slight difference at low frequencies in the prediction for both the shell displacements and the interior pressure and the Maestrello and Efimtsov models exhibit even less sensitivity. Results also indicate that the hydrodynamic coincidences are not sensitive to the choice of the convected velocity of the flow for these particular parameters.

Figures 6 and 7 show a comparison of the NASD between the double shells and single shells for $M=$ 1.42. In these figures, double refers to the system in Fig. 1 and outer and inner refer to either a single outer shell or a single inner shell excited by the boundary turbulence, respectively. For structural response, the double shell system does not offer significant improvement over a single outer shell. The increased response of the "inner" case is due to the reduced weight implicit in the inner panel construction. However, for the interior sound pressure level, the double shell system provides at least $20 \mathrm{~dB}$ reduction relative to the single shell system. If the total mass of the inner shell is added to the system of the "outer", the study of this problem becomes to a comparison between two systems which have the same weight. The authors have made an investigation of this problem in a previous study [13].

\section{Concluding Remarks}

An analytical method is presented that predicts the normalized average spectral density of the response and the interior noise level of two concentric cylindrical sandwich shells excited by the TBL pressure fluctuations. The simulation of the pressure field due to the TBL is taken from previously developed methods by Corcos, Maestrello, and Efimtsov. Classical thin shell theory and the first-order shear deformation theory are applied to the vibration of the inner and outer shells, respectively. A modal expansion technique and Galerkin approach are used to derive closed forms of the shell displacements and the acoustic pressures. Parametric studies are carried out for both subsonic and supersonic flows and for the choices of the convected velocity. Results are discussed among these models and comparisons are made between double and single shells. The following conclusions can be drawn:

(1). The prediction of the normalized average spectral density levels for the structural responses and the interior pressure converges at high frequency for the three TBL models (Corcos, Maestrello, and Efimtsov) examined. 
(2). The normalized average spectral density of the interior pressure is affected not only by the hydrodynamic and acoustic coincidences, but also strongly by the resonances modes above the hydrodynamic and acoustic coincidences at which noise can be transmitted efficiently.

(3). The choice of the convective velocity of the flow does not lead to a significant difference in overall structural response or the interior pressure.

(4). The double wall system provides a significant noise reduction in the interior space over single wall systems although the structure is not noticeably reduced compared to the single wall system.

\section{Reference}

1. Blake, William K., "Mechanics of flow-induced sound and vibration", Vol. I and II, Academic Press, Inc., 1986.

2. Corcos, G. M., " Resolution of pressure in turbulence", J. Acoust. Soc. Am., Vol. 35(2), 1963, pp. 192-199.

3. Dowell, E. H., "Transmission of noise from a turbulent boundary layer through a flexible plate into a closed cavity", J. Acoust. Soc. Am., Vol. 46(1), 1969, pp. 238-252.

4. Efimtsov, B. M., "Characteristics of the field of turbulent wall pressure fluctuations at large Reynolds numbers", Sov. Phys. Acoust., Vol. 28, No. 4, 1982, pp. 289-292.

5. Ffowcs Williams, J. E., "Boundary-layer pressure and the Corcos model: a development to incorporate low-wavenumber constraints", $J$. Fluid. Mech, Vol. 125, 1982, pp. 9-25.

6. Graham, W. R., "A comparison of models for the wavenumber-frequency spectrum of turbulent boundary-layer pressures", The CEAS/AIAA $1 s t$ Joint Aeroacoustic Conference, Munich, Germany, Jun. 12-15, 1995, pp. 711-720.

7. Greespon, J. E., "Random loading and radiation from stiffness and sandwich type cylindrical shell structures", J. Res. Assoc. Tech., Report. No. 7, Sept, 1962.

8. Hwang, Y. F. and Maidanik, G., "A wavenumber analysis of the coupling of a structural mode and flow turbulence", J. of Sound Vib. , Vol. 142(1), 1990, pp. 135-152.

9. Maestrello, L., " Radiation from and panel response to a supersonic turbulent boundary", $J$. Sound. Vib., Vol. 10(2), 1969, pp. 261-295.

10. Norton, M. P., "Fundamentals of noise and vibration analysis for engineers", Cambridge University Press, 1989.

11. Powell, Alan, "On the fatigue failure of structures due to vibrations excited by random pressure fields", J. Acoust. Soc. Am., Vol. 30(12), 1958, pp. 1130-1135.
12. Rattayya, J. V. and Junger, M. G., "Flow excitation of cylindrical shells and associated coincidence effects", J. Acoust. Soc. Am., Vol. 36(5), 1964, pp. 878-884.

13. Tang, Y. Y., Robinson, J. H., and Silcox, R. J., "Sound transmission through cylindrical sandwich shell", The 34th AIAA Aerospace Science Meeting and Exhibit, January 15-18, Reno, Nevada, 1996, Paper No. 96-0877.

14. Tang, Y. Y., Silcox, R. J., and Robinson, J. H., "Sound transmission through two concentric cylindrical sandwich shells", The Proceeding of the 14th International Modal Analysis, February 12-15, Dearborn, Michigan, 1996, pp. 14881495.

15. Willmarth, W. W. and Wooldridge, C. E., "Measurements of the fluctuating pressure at the wall beneath a thick turbulent boundary layer", University of Michigan, ORA Rept. 02920-1-T, 1962.

16. White, P. H., "Transaction of boundary-layer Noise by a rectangle panel", J. Acoust. Soc. Am., Vol. 40(6), 1966, pp. 1354-1362. 
Table 1. Material properties of the face sheet of the sandwich shells.

\begin{tabular}{|l|c|}
\hline \multicolumn{2}{|c|}{ titanium } \\
\hline mass density: $\rho_{1}^{s}\left(\mathrm{~kg} / \mathrm{m}^{3}\right)$ & 4510 \\
\hline elastic modulus: $E(\mathrm{GPa})$ & 120.02 \\
\hline Poison's ratio: $\mu$ & 0.361 \\
\hline \multicolumn{2}{|c|}{ graphite/epoxy layer } \\
\hline mass density: $\rho_{2}^{s}\left(\mathrm{~kg} / \mathrm{m}^{3}\right)$ & 1580 \\
\hline elastic modulus: $E_{\alpha}(\mathrm{GPa})$ & 181 \\
\hline elastic modulus: $E_{\beta}=E_{z}(\mathrm{GPa})$ & 10.3 \\
\hline shear modulus: $G_{\alpha \beta}=G_{z \alpha}(\mathrm{GPa})$ & 7.17 \\
\hline shear modulus: $G_{\beta z}(\mathrm{GPa})$ & 2.87 \\
\hline Poison's ratio: $\mu_{\beta \alpha}$ & 0.33 \\
\hline Poison's ratio: $\mu_{z \alpha}=\mu_{z \beta}$ & 0.28 \\
\hline
\end{tabular}

Table 2. Equivalent material properties of the honeycomb core.

\begin{tabular}{|l|c|}
\hline \multicolumn{2}{|c|}{ titanium honeycomb core } \\
\hline mass density: $\rho_{1}^{s}\left(\mathrm{~kg} / \mathrm{m}^{3}\right)$ & 520.77 \\
\hline elastic modulus: $E_{z}=E_{\epsilon}(\mathrm{GPa})$ & 0.277 \\
\hline elastic modulus: $E_{\zeta}(\mathrm{GPa})$ & 120.02 \\
\hline shear modulus: $G_{z \zeta}=G_{\theta \zeta}(\mathrm{GPa})$ & 2.545 \\
\hline shear modulus: $G_{z \theta}(\mathrm{GPa})$ & 0.069 \\
\hline Poison's ratio: $\mu_{z \theta}$ & 1 \\
\hline Poison's ratio: $\mu_{z \zeta}=\mu_{\theta \zeta}$ & 0.361 \\
\hline \multicolumn{2}{|c|}{ aluminum honeycomb core } \\
\hline mass density: $\rho_{2}^{s}\left(\mathrm{~kg} / \mathrm{m}^{3}\right)$ & 317.54 \\
\hline elastic modulus: $E_{z}=E_{6}(\mathrm{GPa})$ & 0.166 \\
\hline elastic modulus: $E_{\zeta}(\mathrm{GPa})$ & 72 \\
\hline shear modulus: $G_{z \zeta}=G_{\theta \zeta}(\mathrm{GPa})$ & 1.599 \\
\hline shear modulus: $G_{z \theta}(\mathrm{GPa})$ & 0.042 \\
\hline Poison's ratio: $\mu_{z \theta}$ & 1 \\
\hline Poison's ratio: $\mu_{z \zeta}=\mu_{\theta \zeta}$ & 0.3 \\
\hline
\end{tabular}

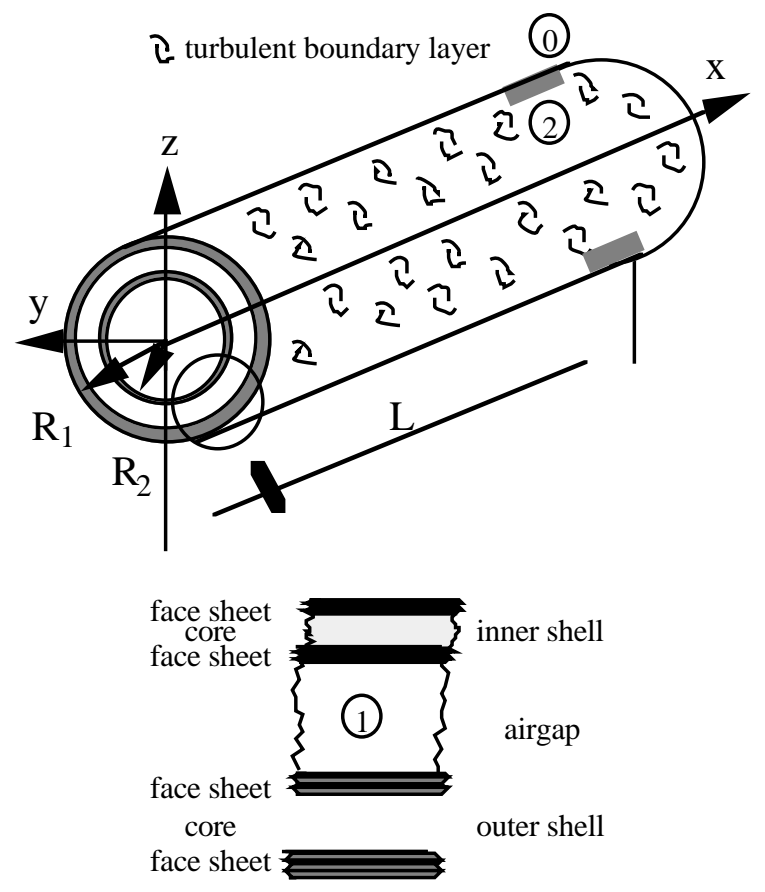

cross section of the shells

Figure 1. A schematic of the system configuration. 

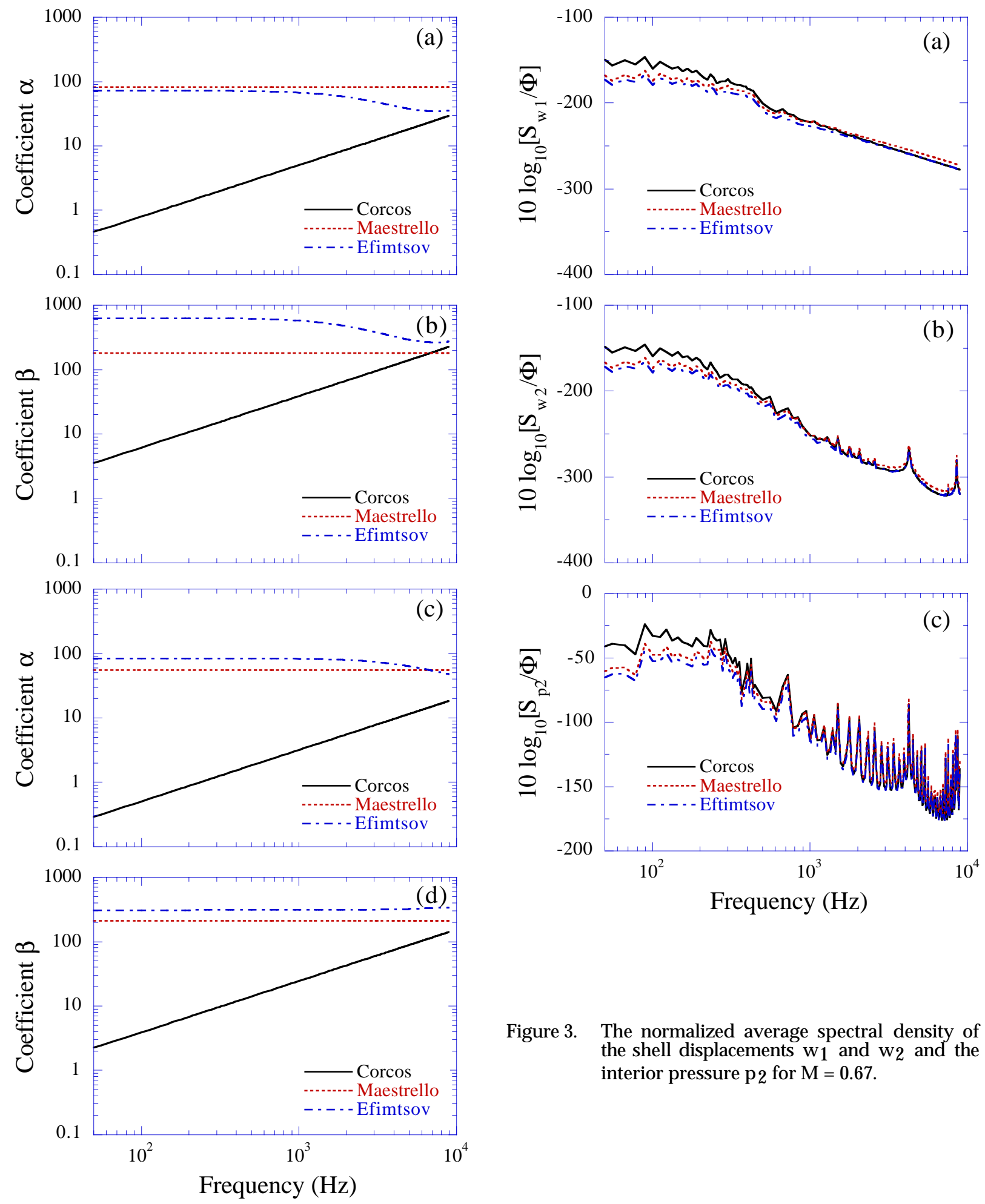

Figure 3. The normalized average spectral density of the shell displacements $\mathrm{w}_{1}$ and $\mathrm{w}_{2}$ and the interior pressure $\mathrm{p}_{2}$ for $\mathrm{M}=0.67$.

Figure 2. The exponential coefficients $\alpha$ and $\beta$ :
(a) and (b) for $\mathrm{M}=0.67$;
(c) and (d) for $\mathrm{M}=1.42$. 

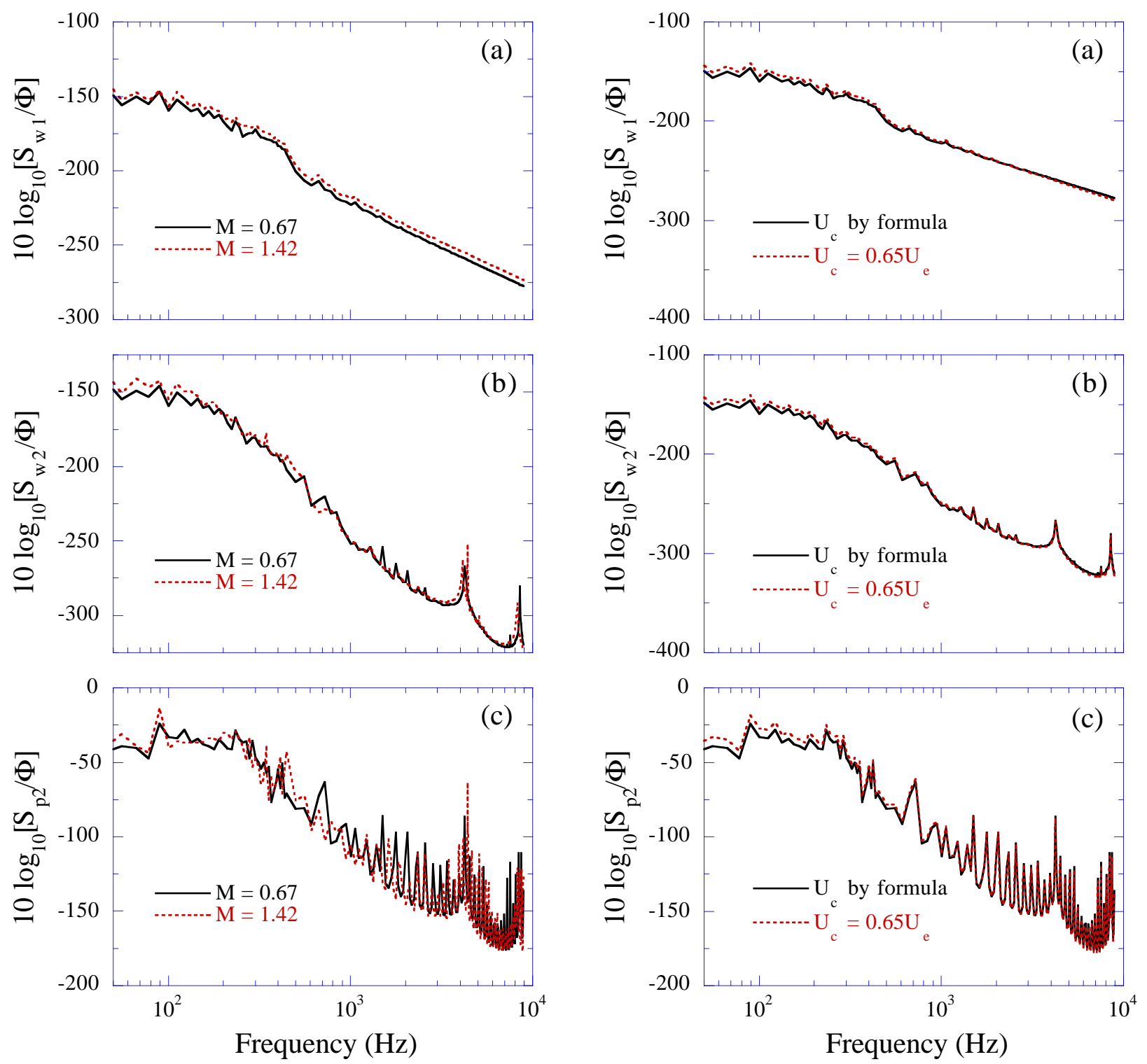

Figure 4. The effect of the Mach number on the normalized average spectral density of the shell displacements $\mathrm{w}_{1}$ and $\mathrm{w}_{2}$ and the interior pressure $\mathrm{p}_{2}$ for Corcos model.

Figure 5. The effect of the choice of $U_{C}$ on the normalized average spectral density of the shell displacements $\mathrm{w}_{1}$ and $\mathrm{w}_{2}$ and the interior pressure $\mathrm{p}_{2}$ for $\mathrm{M}=0.67$ for Corcos model. 

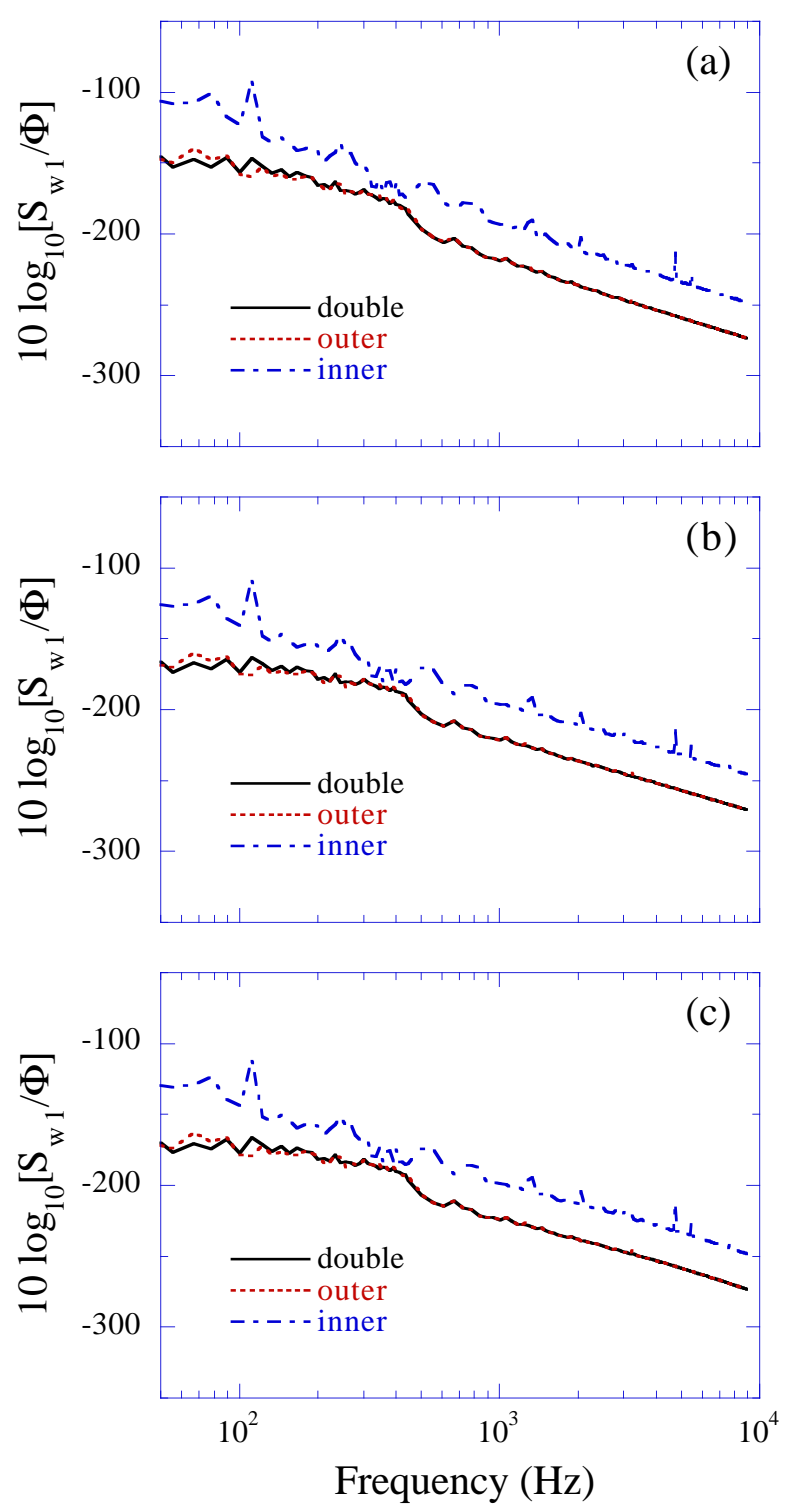

Figure 6. Comparison of the normalized average spectral density of the shell displacement w1 among the double shells, the single outer shell, and the single inner shell for $M=1.42$ : (a). Corcos; (b). Maestrello; (c). Efimtsov.
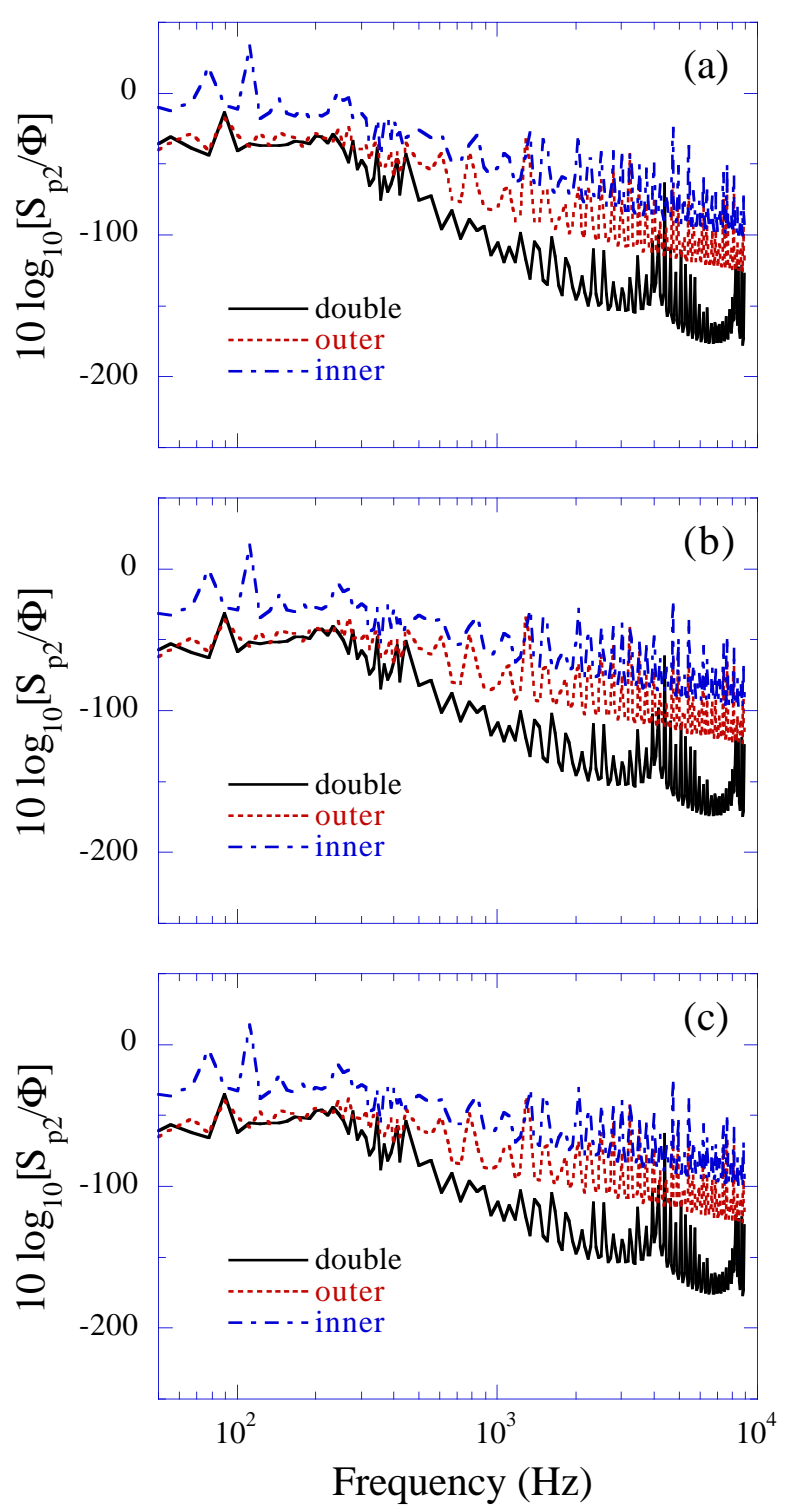

Figure 7. Comparison of the normalized average spectral density of the interior pressure p2 among the double shells, the single outer shell, and the single inner shell for $\mathrm{M}=1.42$ : (a) Corcos; (b) Maestrello; (c) Efimtsov. 